\title{
Observational Study to Assess Quality of Life in Patients with Pancreatic Neuroendocrine Tumors Receiving Treatment with Everolimus: The OBLIQUE Study (UK Phase IV Trial)
}

\author{
John K. Ramage ${ }^{a}$ Pankaj Punia ${ }^{b}$ Olusola Faluyi ${ }^{c}$ Andrea Frilling ${ }^{d}$ \\ Tim Meyer $^{\mathrm{e}}$ Ruby Saharan ${ }^{\mathrm{f}}$ Juan W. Valleg \\ ${ }^{a}$ Kings College Hospital, London and Hampshire Hospitals, London, UK; ${ }^{b}$ Queen Elizabeth Hospital, Birmingham, \\ UK; ' Clatterbridge Cancer Centre, The Wirral, UK; ${ }^{\mathrm{d} I m p e r i a l ~ C o l l e g e ~ L o n d o n, ~ L o n d o n, ~ U K ; ~}{ }^{\mathrm{e}}$ Royal Free Hospital, \\ London, UK; ${ }^{\mathrm{f}}$ Novartis Pharmaceuticals Ltd., Camberley, UK; ${ }^{9}$ University of Manchester, Division of Cancer Sciences/ \\ The Christie NHS Foundation Trust, Manchester, UK
}

\section{Keywords}

Health-related quality of life · Pancreatic neuroendocrine tumours · Everolimus · Real-world study

\begin{abstract}
Background/Aims: To assess health-related quality of life (HRQoL), treatment patterns, and clinical outcomes of adult ( $\geq 18$ years) patients with advanced (unresectable or metastatic) pancreatic neuroendocrine neoplasms (PanNENs) treated with everolimus in routine clinical practice. Methods: In a prospective, non-interventional, multi-center study patients administered at least one $10 \mathrm{mg}$ dose of everolimus were evaluated for change in HRQoL (EORTC QLQ-C30 Global Health Status scale) from baseline after 6 months treatment (primary endpoint). Secondary endpoints included disease-specific HRQoL measures (EORTC QLQ-G.I.NET21), clinical outcomes, everolimus treatment patterns, and safety. Results: Forty-eight patients were recruited (between August 2013 and March 2015); the median treatment duration was 27.8 months. EORTC QLQ-C30 Global Health score was not significantly different from baseline after 6 months of treatment (mean difference -1.9 points, $p=0.660, n=30$ ). In pairwise analyses, the only significant changes in $\mathrm{HRQ}$ oL
\end{abstract}

from baseline were for EORTC QLQ-C30 physical functioning score at month 3 (adjusted mean difference -8.8 points, $p=$ $0.002, n=36$ ) and the EORTC QLQ-G.I.NET21 disease-related worries scores at months 1 and 2 (adjusted mean differences: -11.5 points $[p=0.001, n=44]$ and -8.8 points $[p=0.017$, $n=43]$, respectively). Disease progression or death was recorded in $44.4 \%$ ( $n=20 / 45)$ patients during follow-up; median progression-free survival was 25.1 months and the cumulative survival rate at 3 years was $71 \%$. No new safety signals were detected. Conclusions: The OBLIQUE study demonstrates that HRQoL is maintained in patients with PanNENs during treatment with everolimus in a UK realworld setting. This study adds to the limited HRQoL data available in this patient group.

๑) 2019 S. Karger AG, Basel

\section{Introduction}

Pancreatic neuroendocrine neoplasms (PanNENs) comprise less than $10 \%$ of all neuroendocrine neoplasms (NENs); their annual incidence is estimated to be $0.1-0.2$ per 100,000 population with peak occurrence between ages 40 and 60 years $[1,2]$.

\section{KARGER}

(c) 2019 S. Karger AG, Basel

E-Mail karger@karger.com

www.karger.com/nen 
Although PanNENs are sometimes considered to be indolent, patients with unresectable or metastatic tumors have a lethal disease, and all PanNENs, with the exception of $90 \%$ of insulinomas, have long-term metastatic potential. Morbidity can arise both from secreted hormones and tumor bulk [3]. The grading and staging of PanNENs follows the World Health Organization (WHO 2010 and the more recent WHO 2017), European Neuroendocrine Tumor Society and American Joint Committee on Cancer/ Union for International Cancer control staging systems [4-6].

Effective drug treatment options for advanced PanNENs have historically been limited. The recent approval of sunitinib and everolimus in the UK has helped to fulfil unmet treatment needs. Recent 2017 NICE (UK National Institute for Health and Care Excellence) guidelines recommend everolimus and sunitinib as options for treating well- or moderately-differentiated unresectable or metastatic PanNENs in adults with progressive disease [7]. Sunitinib is a vascular endothelial growth factor inhibitor [8], and everolimus is the first mammalian target of rapamycin inhibitor available for the treatment of this disease [9].

Although survival rates have improved for patients with metastatic PanNENs, advanced NENs in general are still associated with a substantial health-related quality of life (HRQoL) burden, resulting from both the disease itself and treatment-related adverse events (AEs) [10]. Despite this, there is a paucity of HRQoL studies [11, 12], and the translation of HRQoL findings into clinical practice is hampered by the use of multiple HRQoL assessment tools, the use of only generic HRQoL tools in some studies, and a lack of comparative data between treatments [12]. As a result, recommendations have been made that future studies include both a generic HRQoL survey and a NEN-specific survey [12]. Currently, no real-world studies of HRQoL in patients with NENs have been reported in the UK.

The purpose of this prospective, non-interventional UK study was to assess HRQoL, treatment patterns, and clinical outcomes of patients with advanced PanNENs receiving oral treatment with everolimus in routine clinical practice.

\section{Patients and Methods}

\section{Study Design and Patients}

This was a prospective, non-interventional, and multi-center, post-marketing (phase IV) study of adult patients with advanced progressive PanNENs receiving oral treatment with everolimus 10 $\mathrm{mg}$ o.d. in routine clinical practice. There were no imposed diagnos- tic or therapeutic interventions on patients. This study was approved by the south central Southampton research Ethics Committee, reference number 13/SC/0121. Patients were recruited from 14 centers in the UK (13 in England and 1 in Scotland) between August 2, 2013 and March 3,2015. The participating centers were selected according to the following criteria: recognized European Neuroendocrine Tumor Society Centers of Excellence, expected availability of sufficient study participants, NEN expert multidisciplinary team. Patients were enrolled into the study after providing written informed consent, and when all study eligibility criteria had been confirmed: aged $\geq 18$ years with a diagnosis of advanced (unresectable or metastatic, confirmed histologically); well-differentiated and/or grade 1/2 PanNENs with radiologically documented progressive disease, and a decision made to start once-daily treatment with $10 \mathrm{mg}$ everolimus. Patients were excluded from the study if their medical history contraindicated a recommendation for everolimus treatment [13].

All screening evaluations were performed within one month prior to everolimus initiation, according to normal clinical practice, and patients were assessed as being suitable to start treatment with everolimus prior to study entry. The baseline period was up to 1 month before the start of treatment, during which time the necessary administrative steps (unrelated to this study) were taken to enable patients to start everolimus treatment. Patients continued to receive everolimus until a clinical decision was made to stop therapy. $\mathrm{Pa}$ tients were followed from the time of written informed consent until 1 month after the last dose of everolimus, until early termination (voluntary patient withdrawal, clinician decision to withdraw patient on clinical grounds, patient lost to follow-up), or until the end of the study, whichever was sooner (observation period).

\section{Primary and Secondary Endpoints}

The primary endpoint of this study was change in HRQoL, as assessed by the European Organization for Research and Treatment for Cancer Quality of Life Questionnaire (EORTC QLQC30, Global Health Status score) after 6 months of oral treatment with everolimus $10 \mathrm{mg}$ o.d. compared to baseline.

The EORTC QLQ-C30 is a 30 -question, self-administered questionnaire with 9 multiple-item scales, designed to assess HRQoL in oncology patients; the Global Health Status and QoL scale is one of the item scales [14].

The secondary endpoints were changes in HRQoL using the EORTC QLQ-C30, EORTC QLQ-GI.NET21, and EQ-5D questionnaires, at approximately $1,2,3,4,5$, and 6 months following everolimus initiation.

The EORTC QLQ-GI.NET21 is a 21-question, self-administered questionnaire designed to assess HRQoL in patients with gastrointestinal (GI)-related NENs, who vary in disease stage and treatments. This questionnaire has 3 multi-item symptom scales (endocrine: 3 questions, GI: 5 questions, treatment-related side effects: 3 questions), 2 single item symptoms (bone/muscle pain, concern about weight loss), 2 psychosocial scales (social function: 3 questions, disease-related worries: 3 questions), and 2 other single items (sexuality and communication) [15].

The 6-item EQ-5D self-administered questionnaire, designed to assess health status in adults, comprises 2 distinct components: the first addresses domains of mobility, self-care, usual activity, pain/discomfort, and anxiety/depression and the second measures self-rated (global) health status using a visual analog scale [16].

Additional secondary endpoints included: progression-free survival (PFS) on the basis of radiological and/or clinical findings 
assessed according to routine clinical practice at participating centers, overall survival (OS), changes in biochemical tumor markers (chromogranin A, and other makers, for example, insulin, gastrin, glucagon, as appropriate) at approximately 1, 2, 3, 4, 5, and 6 months of everolimus treatment; duration of everolimus treatment; and the overall safety and tolerability of everolimus.

\section{Definition of Dose Changes}

Dose interruptions during the analysis period were defined as dose reduced to $0 \mathrm{mg}$ /day for a period greater than 1 day and subsequently restarted. Dose reductions were defined as a reduction in everolimus dose from the previous cycle followed by a subsequent increase in dosage.

\section{Data Collection}

In this real-world setting, the usual standard of care was for patients to be seen monthly for the first 6 months, followed by visits approximately every 4-8 weeks. Patients completed the 3 HRQoL questionnaires at baseline, and each time they visited the clinic in the first 6 months of everolimus treatment. Completion of HRQoL questionnaires was the only study-specific procedure and data for all other study outcomes were collected as recorded in the patients' medical records at each follow-up visit.

Details of baseline patient demographic and clinical characteristics (including prior and concomitant medications and non-drug therapies [e.g., surgery, peptide receptor radionuclide therapy]) and data related to everolimus treatment, efficacy, and safety were recorded during the observation period. Data were collected from patients' medical records and entered into electronic case report forms, which were validated and reviewed for completeness and accuracy.

AEs and serious AEs (SAEs) were collected throughout the study and for 1 month after the last dose of everolimus. An AE was defined as any untoward medical occurrence in a patient included in this study. An SAE was defined as an event which was fatal or life-threatening or resulted in persistent or significant disability/incapacity or constituted a congenital anomaly/birth defect or required unplanned inpatient hospitalization or prolongation of hospitalization or was medically significant or involved transmission of infectious agent via medicinal product. AEs were coded using the medical dictionary for regulatory activities terminology and were designated as either related or unrelated to treatment by the clinical investigator. Special safety assessments (weight, blood pressure, heart rate, clinical chemistry, lipid profile, hematology, coagulation, urine analysis, and ECG assessments) were collected throughout the study, and for 1 month after the last dose of everolimus, where available.

The efficacy, safety, and treatment data were initially planned to be collected up to 36 months after everolimus initiation. However, since the primary endpoint (HRQoL assessments 6 months after initiation of everolimus) had been met and the study sample size was above the original target of 33 patients, a decision was made by the sponsor and chief investigator and the study was completed earlier than originally projected. The median time of followup was 22.0 (interquartile range [IQR] 14.5-29.5) months.

\section{Statistical Analysis}

Sample Size

Sample size requirements were estimated using a normal approximation as Global Health Status QoL score is one of the relatively few scales of the EORTC QLQ-C30 that is often approximately normally distributed. No reference data for patients with ad- vanced NETs is presented in the EORTC QLQ-C30 manual; as a consequence the reference value for "all cancer patients: recurrent/ metastatic" was used (mean Global Health Status score 56.3 [SD $25.6]$ ) to provide an approximate comparable sample in terms of the severity of disease. It was considered that a change in score of $0.5 \mathrm{SD}$ would provide a minimum value for an important patient-perceived change. Assuming an SD of 25.6, a study sample size of 30 would have $75 \%$ power and a study sample size of 40 would have $86 \%$ power to detect a change from baseline of 12.8 points in Global Health Score based on a single group $t$ test with a 0.05 two-sided significance level. At the start of the study, a minimum sample size of 33 was planned to allow for the possibility of up to $10 \%$ of patients failing to complete the first 6 months of treatment. Sample size calculations were performed with NQuery Advisor 7.0.

\section{Definition of Patient Populations}

The safety set population was defined as all patients who received at least one dose of everolimus and had at least one postbaseline safety assessment; this population was used for analysis of everolimus exposure and safety. The full analysis set (FAS) population consisted of patients who received at least one dose of everolimus and completed at least one questionnaire in the first 6 months of treatment (post-baseline efficacy assessment); this population was used for analysis of all other study outcomes.

\section{Data Analysis}

Data were analyzed using the statistical package STATA version 14 (StataCorp. LP, College Station, TX, USA). Descriptive statistics including mean (SD), median (IQR) for continuous variables, and frequencies and percentages for categorical variables are reported. The change from baseline in EORTC QLQ-C30 after 6 months of treatment with everolimus was assessed by paired $t$ test. Changes in EORTC QLQ-C30, EORTC-QLQ-GI.NET21, and EQ5D-5L scores from baseline at the monthly post-initiation assessment visits (Months 1, 2, 3, 4, 5, and 6) was assessed by univariate analysis of variance for repeated measures. If a significant effect across time points in the univariate analysis of variance model was observed, pairwise comparisons between baseline and the different time points were calculated using a correction factor for multiple testing (Dunnett pair-wise calculation). Results were considered significant at $p \leq 0.05$.

\section{Analysis of Time-Dependent Variables}

Time-dependent variables were analyzed using the Kaplan-Meier method and reported as median $95 \% \mathrm{CI}$ and/or estimated cumulative survival (\%) at 1,2, and 3 years following everolimus initiation. OS was defined as the time from everolimus initiation until death from any cause; patients not recorded as having died at the end of the study were censored at the last date they were known to be alive. PFS was defined as the time from everolimus initiation to disease progression (based on the investigator's assessment of the clinical and/or radiological findings) or death; patients not documented as having progressive disease or died (from any cause) were censored on the date of the last disease assessment of known severity.

The duration of everolimus treatment was defined as the time from everolimus initiation to treatment discontinuation (any temporary discontinuations were not taken into account in assessing treatment duration); patients not documented as discontinuing treatment were censored on the date they were last recorded as receiving treatment. 


\section{Results}

\section{Patient Population and Demographics}

Forty-eight patients (range 1-8 per center) met the eligibility criteria and were included in the study (online suppl. Fig. S1; for all online suppl. material, see www. karger.com/doi/10.1159/000497330). The study data collection period ran from August 2013 to December 2016. All 48 patients were included in the safety set population; 46 patients were included in the FAS population. Of the 48 patients in the safety set, the number of patients being observed at months $1,2,3,4,5$, and 6 assessment visits were $47,46,43,41,38$, and 35 , respectively. Of the 46 patients in the FAS population, the number of patients being observed at months $1,2,3,4,5$, and 6 assessment visits were $46,46,43,41,38$, and 35 , respectively. The baseline demographics and clinical characteristics of patients included in the FAS are summarized in Table 1. The median age of patients at diagnosis was 60.0 years and at study entry was 64.3 years, 32/46 (70\%) of study participants were men, and 14/46 (30\%) of participants had an ECOG performance status of 0 at baseline. A total of 33/46 (72\%) of patients had metastatic disease documented at baseline (online suppl. Table S1); 24/46 (52\%) of patients had a G2 WHO 2010 tumor grading with a mean (SD) Ki67 index score of $10.4(8.7 ; n=20$, online suppl. Table S2).

The therapies received prior to everolimus initiation are summarized in online supplementary Tables S3 and S4; $35 \%$ of patients $(n=17 / 48)$ had received only one prior anti-cancer treatment before initiation of everolimus.

\section{Treatment with Everolimus}

The mean everolimus dose in the safety set population remained relatively stable throughout months 1-6 postinitiation period (Fig. 1).

\section{Treatment Changes}

At least one temporary dose interruption during the observation period was documented for 29/48 (60.4\%) patients, with a mean of 1.4 (SD 1.6) interruptions per patient ( $n=47$, exposure data were not available for 1 patient) and mean duration of interruptions of 17.5 days (SD 15.8, $n=66$ interruptions). At least one dose reduction was documented for 40/48 (83.3\%) patients, with a mean of 2.3 (SD 1.8) reductions per patient ( $n=47$, exposure data were not available for 1 patient). The majority of patients had at least one change in treatment regimen (dose reductions, interruptions, and discontinuations) and the first change in treatment occurred in 24/48 (50\%) of patients within 2 months of everolimus initia-
Table 1. Patient demographics and clinical characteristics at baseline (FAS population)

\begin{tabular}{lc}
\hline Patient demographics & $\begin{array}{l}\text { FAS population } \\
(n=46)\end{array}$ \\
\hline Age at diagnosis, years $^{\mathrm{a}}$ & $60.0(38.1-77.9)$ \\
Age at study entry, years $^{\mathrm{a}}$ & $64.3(39.7-78.5)$ \\
Gender, male & $32(70)$ \\
Ethnicity & \\
$\quad$ Caucasian & $40(87)$ \\
Asian & $3(7)$ \\
Unknown & $3(7)$ \\
Performance status, ECOG & \\
0 & $14(30)$ \\
1 & $19(41)$ \\
2 & $4(9)$ \\
Not assessed & $9(20)$ \\
\hline
\end{tabular}

Data presented as: ${ }^{\mathrm{a}}$ median (range), ${ }^{\mathrm{b}} n(\%)$. ECOG, Eastern Cooperative Oncology Group; FAS, full analysis set.

tion (Fig. 2a). A total of 23/48 (48\%) of patients discontinued everolimus treatment during the study observation period and the most frequent reason for treatment discontinuation was AEs (11/23 [48\%], online suppl. Table S5). The median cumulative treatment duration was 27.8 months (95\% CI 7.8 to NR [upper CI not reached]) and the cumulative proportions of patients remaining on treatment at 1,2, and 3 years following initiation were $63 \%$ (95\% CI $47-76 \%$ ), 51\% (95\% CI 34-65\%), and 19\% (95\% CI 2-50\%), respectively.

\section{Health-Related Quality of Life}

EORTC QLQ-C30 (Global Health Status QoL Score)

For the 30 patients in the FAS population with Global Health Status scores available at baseline and also at month 6 assessment visit, no significant difference in EORTC QLQ-C30 score was observed at month 6 (mean score 55.0 [95\% CI 48.1-61.9]) compared with baseline (mean score 56.9 [95\% CI 50.3-63.6]; mean change - 1.9 [95\% CI - 10.9$7.0]$ points, $p=0.660$ ). There were no significant differences in Global Health Status between baseline and any assessment visit following everolimus initiation (Table 2; Fig. 3) when all patients with paired scores were evaluated. A post-hoc analysis was carried out to evaluate changes in EORTC QLQ-C30 Global Health Status scores during the first 6 months of treatment in the subgroup of patients continuing on everolimus at month $6(n=30)$; no significant differences were observed between baseline and any assessment visit following everolimus initiation (Table 3). 
Fig. 1. Mean (95\% CI) dose of everolimus (mg/day) at each study assessment visit following start of initial $10 \mathrm{mg}$ dose (safety set population, $n=48$ ).

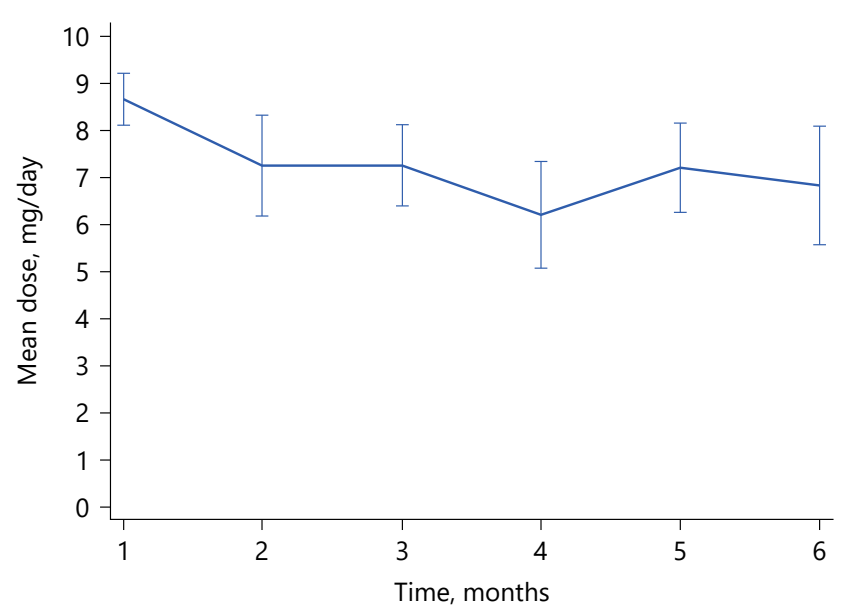

Median: 1.9

(95\% Cl: 0.8-3.0)

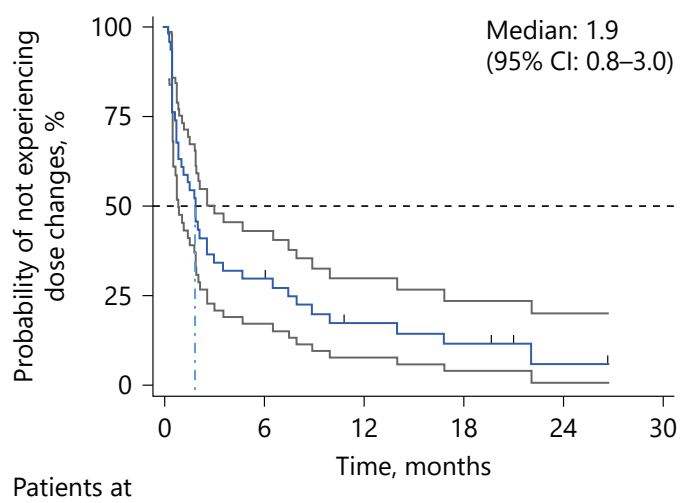

a risk 46

7

0

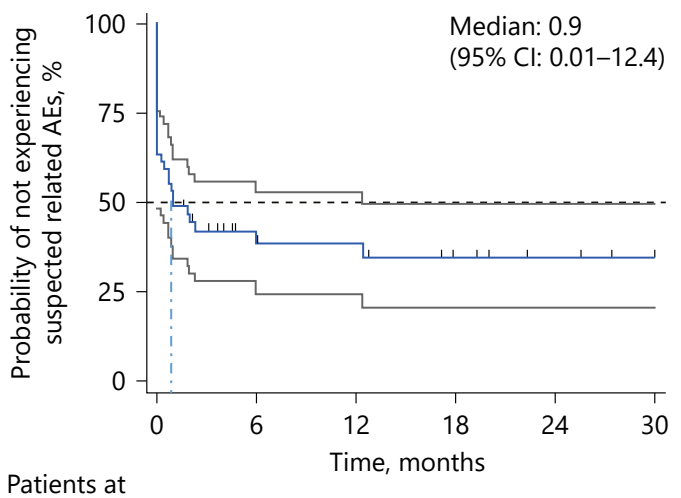

b risk 47
10
Fig. 2. Everolimus treatment and suspected related AEs (safety set population, $n=48$ ). a Kaplan-Meier curve showing the probability (95\% confidence limits and median months) of patients remaining without everolimus dose changes (dose reductions, inter-

In the overall sample, a significant difference from baseline in EORTC QLQ-C30 physical functioning scale score during the first 6 months of everolimus treatment was observed (online suppl. Table S6). In pairwise analyses, only the score at month 3 was significantly different from baseline (adjusted mean difference -8.8 [95\% CI -15.1 to -2.5 ] points, $p=0.002$ ). There were no significant differences in the other EORTC QLQ-C30 subscales between baseline and any assessment visit dur- ruptions, and permanent discontinuations) during follow-up. b Kaplan-Meier curve showing the probability (95\% confidence limits and median months) of patients remaining free from suspected related AEs during follow-up. AE, adverse event. ing the first 6 months of everolimus treatment (online suppl. Table S6).

\section{EORTC QLQ-GI.NET 21 Score}

A significant difference from baseline in EORTC QLQ-GI.NET 21 disease-related worries score during the first 6 months of everolimus treatment was observed, suggesting an improvement (online suppl. Table S7). In pairwise analyses, the only significant differences from base- 


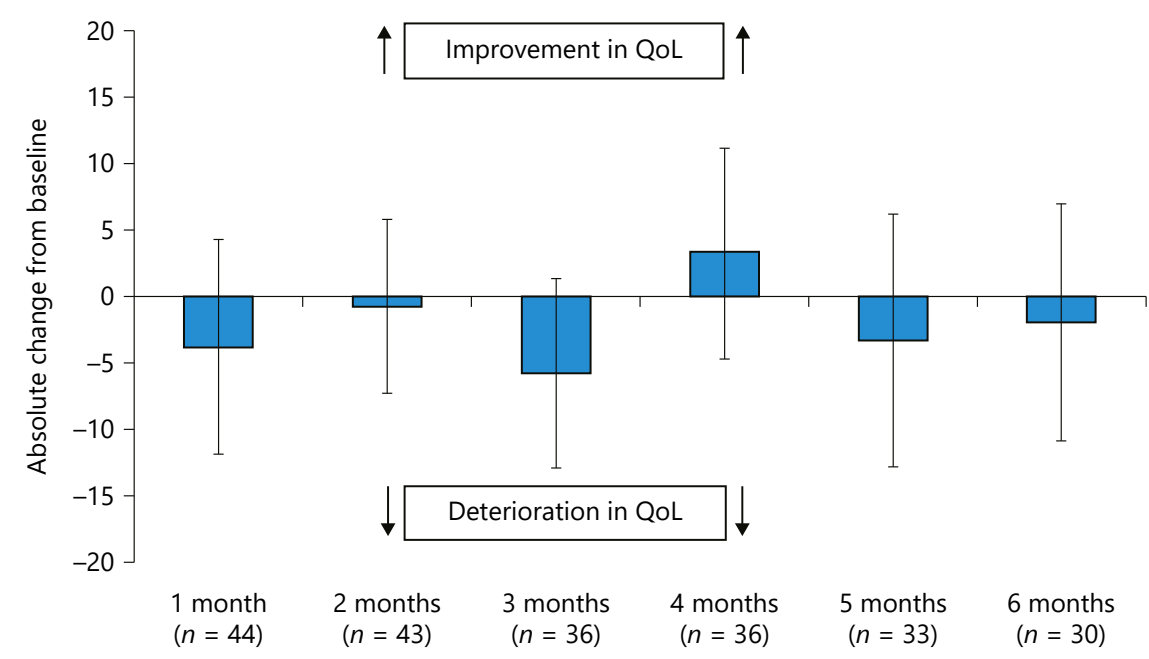

Fig. 3. Absolute change from baseline in EORTC QLQ-C30 Global Health Status score (FAS population, $n=46$ ). An increase in score represents an improvement in HRQoL. FAS, full analysis set; HRQoL, health-related quality of life.

Table 2. Changes from baseline in EORTC QLQ-C30 Global health status during the first 6 months of treatment with everolimus (all patients with paired scores at the specified time points)

\begin{tabular}{|c|c|c|c|c|c|c|}
\hline $\begin{array}{l}\text { EORTC QLQ-C30: } \\
\text { Global health status }\end{array}$ & Month 1 & Month 2 & Month 3 & Month 4 & Month 5 & Month 6 \\
\hline Patients with paired scores, $n$ & 44 & 43 & 36 & 36 & 33 & 30 \\
\hline Baseline score $^{\mathrm{a}}$ & $58.0(20.3)$ & $57.6(20.9)$ & $56.7(20.0)$ & $56.0(19.8)$ & $56.6(19.2)$ & $56.9(17.9)$ \\
\hline $\begin{array}{l}\text { Post-initiation score at } \\
\text { each month }\end{array}$ & $54.2(24.9)$ & $56.8(22.8)$ & $50.9(19.9)$ & $59.3(20.5)$ & $53.3(19.9)$ & $55.0(18.5)$ \\
\hline Absolute change ${ }^{\mathrm{b}}$ & $\begin{array}{l}-3.8 \\
(-11.8 \text { to } 4.3)\end{array}$ & $\begin{array}{l}-0.8 \\
(-7.3 \text { to } 5.7)\end{array}$ & $\begin{array}{l}-5.8 \\
(-12.9 \text { to } 1.3)\end{array}$ & $\begin{array}{l}3.2 \\
(-4.7 \text { to } 11.2)\end{array}$ & $\begin{array}{l}-3.3 \\
(-12.8 \text { to } 6.2)\end{array}$ & $\begin{array}{l}-1.9 \\
(-10.9 \text { to } 7)\end{array}$ \\
\hline Percentage change ${ }^{c}$ & -6.5 & -1.3 & -10.2 & 5.8 & -5.8 & -3.4 \\
\hline
\end{tabular}

Data presented as: ${ }^{\mathrm{a}}$ mean (SD); ${ }^{\mathrm{b}}$ mean $(95 \% \mathrm{CIs}) ;{ }^{\mathrm{c}}$ percentage mean change from baseline.

line were observed at month 1 (adjusted mean difference -11.5 [95\% CI -19.1 to -3.9$]$ points, $p=0.001$ ) and month 2 (adjusted mean difference -8.8 [95\% CI -16.4 to -1.2$]$ points, $p=0.017$ ) and both were suggestive of improvement in scores. There were no significant differences in the other EORTC QLQ-GI.NET 21 subscales between baseline and any assessment visit during the first 6 months of everolimus treatment (online suppl. Table S7).

There were no significant differences in either component of the EQ-5D-5L between baseline and any assessment visit during the first 6 months of everolimus treatment (online suppl. Table S8).

\section{Progression-Free Survival and OS}

Twenty patients in the FAS died or had disease progression during the study, with a median PFS (KaplanMeier analysis) of 25.1 months (95\% CI 7.8 to NR [upper CI not reached]; Fig. 4a), and the cumulative proportion of patients alive and with no evidence of disease progression was 58\% (95\% CI $41-72 \%)$ at 1 year, 51\% (95\% CI $33-66 \%)$ at 2 years, and 35\% (95\% CI 14-56\%) at 35 months (final patient censored by 35 months).

Ten patients died during the study and the median OS was not reached (Fig. 4b); the cumulative proportion of patients alive was $90 \%$ (95\% CI 76-96\%) at 1 year, $71 \%$ 


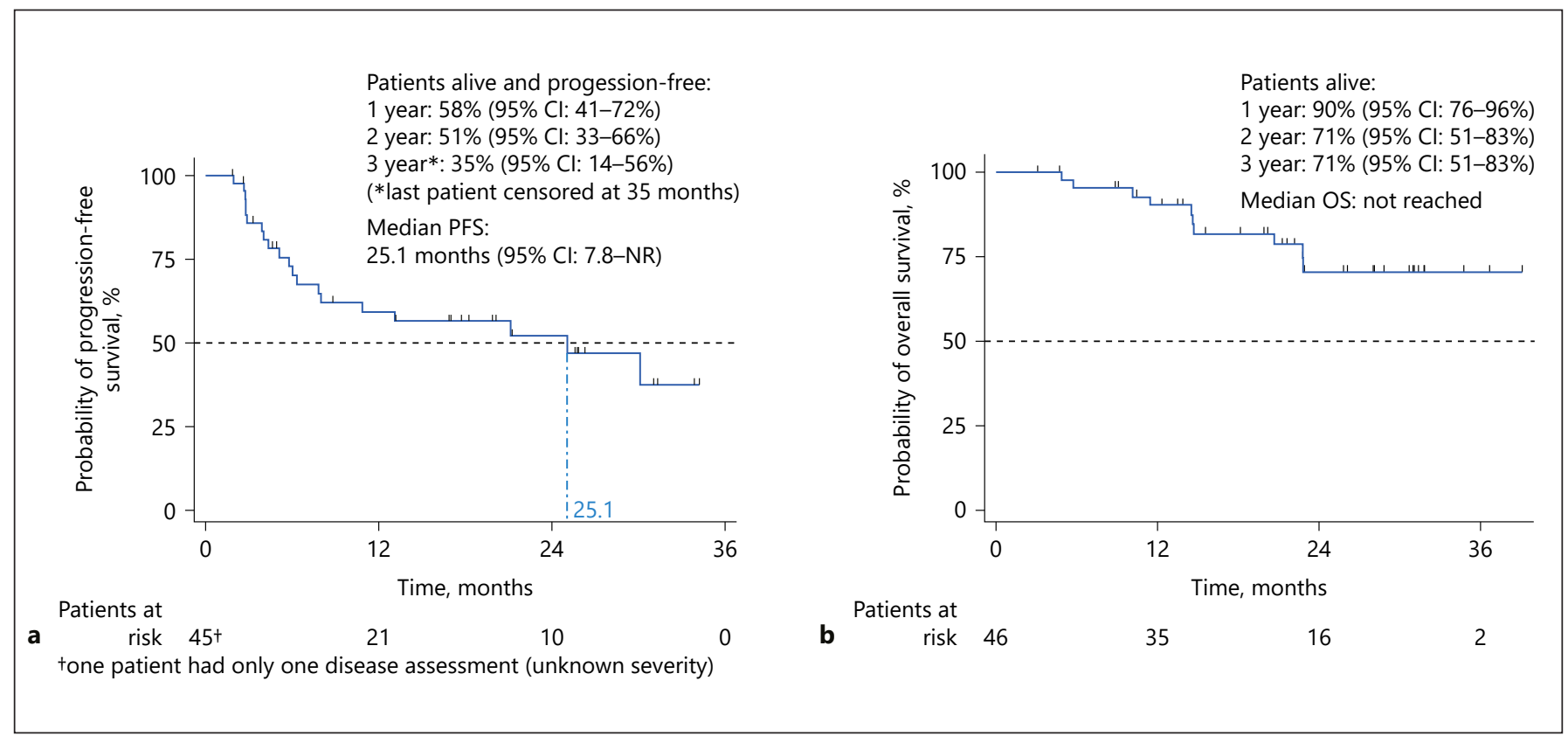

Fig. 4. Progression-free survival and OS (FAS population, $n=46$ ). a Patients alive and progression-free during follow-up. b Patients alive during follow-up. OS, overall survival; FAS, full analysis set.

Table 3. Post-hoc analysis evaluating changes from baseline in EORTC QLQ-C30 Global health status during the first 6 months of treatment with everolimus for the subgroup of $n=30$ patients continuing on everolimus at month 6

\begin{tabular}{lllllll}
\hline $\begin{array}{l}\text { EORTC QLQ-C30: Global health } \\
\text { status }(n=30 \text { continuing treatment } \\
\text { at month 6) }\end{array}$ & Month 1 & Month 2 & Month 3 & Month 4 & Month 5 & Month 6 \\
\hline Patients with paired scores, n $^{\text {Baseline score }}$ & & & & & & \\
Post-initiation score at each month $^{\mathrm{a}}$ & 30 & 29 & 25 & 28 & 28 & 30 \\
Absolute change $^{\mathrm{b}}$ & $56.9(17.9)$ & $56.9(18.2)$ & $56.3(18.2)$ & $56.8(18.0)$ & $58.6(16.7)$ & $56.9(17.9)$ \\
& -3.1 & -2.0 & -5.0 & 2.1 & -2.7 & -1.9 \\
Percentage change $^{\mathrm{c}}$ & $(-13.7-7.6)$ & $(-10.4-6.4)$ & $(-14.0-4.0)$ & $(-7.6-11.7)$ & $(-13.3-7.9)$ & $(-10.9-7.0)$ \\
& -5.4 & -3.5 & -8.9 & 3.7 & -4.6 & -3.3 \\
\hline
\end{tabular}

Data presented as: ${ }^{\mathrm{a}}$ mean $(\mathrm{SD}) ;{ }^{\mathrm{b}}$ mean $(95 \% \mathrm{CIs}) ;{ }^{\mathrm{c}}$ percentage mean change from baseline.

(95\% CI $51-83 \%)$ at 2 years, and $71 \%$ (95\% CI 51-83\%) at 3 years after everolimus initiation.

\section{Safety}

A total of 1,052 AEs in 48 patients were reported (716/1,052 [68.1\%] grade $1 / \mathrm{mild})$, with a median of 19.0 (IQR 14.0-29.5) AEs, and a maximum of 104 AEs, reported per patient. 79/1,052 (7.5\%) of reported AEs were classified as serious, and 33/1,052 (3.1\%) were considered events of special interest. A relationship to everolimus ex- posure was suspected for 468/1,052 (44.5\%) reported AEs. The majority of patients who experienced a suspected related $\mathrm{AE}$ experienced the first suspected related $\mathrm{AE}$ within the first 2 months of everolimus initiation (Fig. 2b). The most frequently reported suspected related AEs were fatigue (41 events in 24 [50.0\%] patients; of these, $n=1$ was grade 3/severe event), rash (28 events in 18 [37.5\%] patients), and mucosal inflammation (32 events in 18 [37.5\%] patients; of these, $n=5$ events were grade $3 /$ severe; Fig. 5). 


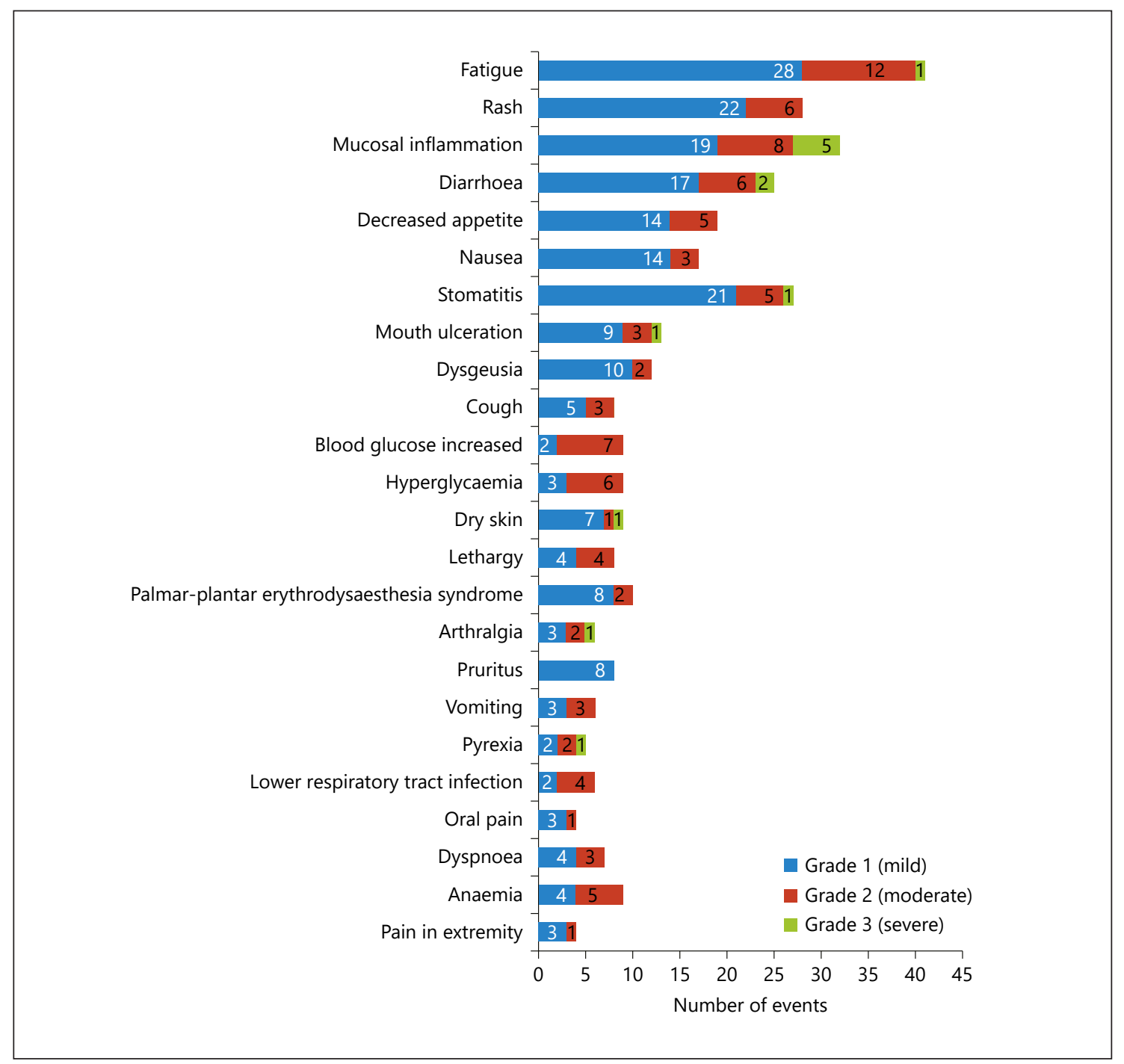

Fig. 5. AEs with a suspected relationship to everolimus observed in $\geq 4$ (8.3\%) patients, stratified by severity. AE, adverse event.

\section{Discussion}

The OBLIQUE study is the first one to evaluate the impact of everolimus on HRQoL in UK patients with well differentiated, grade 1 or grade 2 PanNENs in a realworld setting. The age of patients in this study is similar to that reported in the pivotal RADIANT-3 phase III clinical trial of everolimus [9] and a recent phase IIIb study that evaluated safety and HRQoL in patients with advanced NETs treated with everolimus in an expanded access protocol [17]. About $30 \%$ of patients in our study had a performance status of 0 at baseline, which is lower than the phase III trial (67\%) [9] and expanded access study
(56\%) [17]. This difference may reflect the real-world nature of the OBLIQUE population, with patients limited in their everyday activities to a greater extent than patients included in clinical trials.

It has been previously reported that patients with NETs have worse HRQoL compared with the general population [18], and that treatment-related AEs are one of the factors contributing to poorer HRQoL [10]. For this reason, understanding the impact of therapies on patients' HRQoL (in addition to disease status and tumor burden) is of considerable importance and of particular relevance for everolimus, given the known potential for toxicity. All patients in the present study ex- 
perienced at least one AE, with a median of 19 AEs per patient during the observation period, and $45 \%$ of AEs were suspected to be related to everolimus treatment. Despite this, HRQoL was maintained during the first 6 months of treatment with everolimus, as measured by the EORTC QLQ-C30 Global Health Status score. Although a statistically significant decline in EORTC QLQ-C30 physical functioning score was observed at month 3 assessment and statistically significant declines in EORTC QLQ-GI.NET 21 disease-related worries score were observed at months 1 and 2 assessment visits, these were not sustained. The majority of patients who experienced an AE suspected to be related to everolimus experienced the first event within 2 months of initiation. Similarly, $50 \%$ of patients had a first treatment change within 2 months of initiation, which may have been related to the occurrence of AEs. Therefore, it is possible that the temporary decline in physical functioning and disease-related worries may have been related to the occurrence of treatment-related AEs and the need for dose changes. However, it should be noted that no other statistically significant differences in EORTC QLQ-C30, EORTC QLQ-GI.NET 21 or EQ-5D scores were observed during the first 6 months of everolimus treatment. Taken together these data, therefore, suggest that HRQoL remains maintained throughout the first 6 months of treatment with everolimus in patients with advanced PanNENs.

These results are consistent with those of the recently published Global Expanded Access Study where no clinically significant changes in EORTC QLQ-C30 global health status were observed during a 3-month follow-up period in 86 patients with PanNENs receiving $10 \mathrm{mg}$ o.d. everolimus [17]. A further patient reported outcome study in 44 outpatients (single-center in Italy) with PanNENs receiving either no treatment, or somatostatin analogs (with or without radiometabolic therapy) showed that over a 4-month period, patients had a good physical, but impaired mental component of HRQoL [11]. Direct comparisons with our study are difficult as Pezzilli et al. [11] used different patient reported outcome instruments (and no NET-specific measure) and more than half of their patients were disease-free at the time of the study, potentially accounting for a good physical component. This study adds to the limited HRQoL data available for patients with PanNENs treated with everolimus, and addresses the need for general HRQoL and disease-specific tools to be utilized in HRQoL studies in general and in real-world settings in particular.

Everolimus in Pancreatic Neuroendocrine Tumors
The majority of AEs recorded were grades 1 and 2 (non-serious), emphasizing the importance of effective toxicity management to optimize clinical benefit. No new safety signals were detected, and the safety profile of everolimus was observed to be consistent with the registration study for this population $[9,13]$. Although all 48 patients experienced at least one AE, the median treatment duration was 27.8 months, suggesting that in most cases AEs were well-managed.

The median PFS of 25 months observed in the present study is considerably longer than the 11 months observed in everolimus-treated patients in the RADIANT-3 clinical trial [9]. Greater flexibility in dose modifications are possible in routine clinical practice settings and may have enabled treatment to continue for longer (median treatment duration 27.8 months in OBLIQUE vs. 9 months in RADIANT-3 [9]). The differing definitions of PFS and more stringent assessment of tumor response in the clinical trial may also have contributed to the difference in PFS estimates between the 2 studies. Furthermore, in RADIANT-3, disease progression was documented formally according to RECIST at pre-specified intervals [9] whereas the definition used in this study was based on the investigator's assessment of the clinical and/or radiological findings, and all tests and assessments were in accordance with routine practice.

There were a number of limitations with this study since collection of all post-initiation data were dependent on the frequency of routine hospital visits and management, in accordance with routine practice. HRQoL questionnaires were completed by patients whenever they attended clinic and therefore there was some variability in the timing of the months 1-6 assessment visits post-initiation of everolimus, and the availability of clinical outcomes data were driven by pragmatic clinical assessments. This variability in time of reporting of questionnaires may have affected the precision of measurement of percentage changes in HRQoL scores from baseline. A number of patients discontinued everolimus treatment within 6 months of initiation, which may have led to an overestimate of Global Health Status scores at 6 months since those patients that remained in the study may be those who tolerated the drug well and had good clinical outcomes. A final limitation is that there was no control group with which to compare the results. Although patients acted as their own controls (due to the change from baseline study design), this does not allow separation of treatment effects on HRQoL from disease effects or the confounding effect on HRQoL of investigation results received that an independent control group would have

Neuroendocrinology 2019;108:317-327 325 
provided. The small size of this study and the non-homogenous tumor grading are limitations and further research will be needed for a wider interpretation of these results.

\section{Conclusions}

This is the first real-world study in the UK to demonstrate that HRQoL is maintained in patients with PanNENs receiving everolimus during the first 6 months of treatment, despite the frequent occurrence of suspected related AEs and treatment changes, and supports the results from clinical trials. We also demonstrated that PFS was longer in patients treated in the real-world setting than in the phase III clinical trial, despite having poorer performance status at initiation. Since the study included patients with PanNENs recruited from a range of geographically dispersed UK centers, the results should be generalizable to the wider population of UK patients with this condition. The findings of the present study may also be relevant to the broader population of patients with NENs, although further studies are warranted to support this.

\section{Acknowledgements}

NIHR Manchester Clinical Research Facility for patients treated at The Christie (Manchester). Tim Meyer is supported by NIHR UCH Biomedical Research Centre.

\section{Statement of Ethics}

This study was approved by the south central Southampton research Ethics Committee (Ref. No. 13/SC/0121). The patients provided written informed consent.

\section{Disclosure Statement}

J.K.R. received research grants and meeting expenses from Novartis Pharmaceuticals; P.P. received sponsorship from Novartis for oncology conference; O.F. declares no conflict of interest; A.F. received a research grant, support for travel and speaker honoraria from Novartis; T.M. has been a paid member of an Ipsen Advisory board, declares consulting role for Novartis; R.S. is employed by Novartis; J.W.V. declares Consulting or Advisory Role, Speakers' Bureau and Research Funding from Novartis.

\section{Funding Sources}

This study was sponsored and funded by Novartis Pharmaceuticals UK Limited. Novartis employees were involved in the study design, data analysis, and in the review of this manuscript. $\mathrm{pH}$ Associates, a company specializing in real-world evaluation, was commissioned by Novartis to analyze the study results and support the drafting of this manuscript.

\section{Author Contributions}

J.K.R. contributed to the conception/design of the study, acquisition of data, analysis of data, interpretation of data, review of draft manuscript and approval of final version; P.P. contributed to the conception/design of the study, acquisition of data, analysis of data, interpretation of data, review of draft manuscript, and approval of final version; O.F. contributed to the acquisition of data, analysis of data, interpretation of data, review of draft manuscript, and approval of final version; A.F. contributed to the acquisition of data, analysis of data, interpretation of data, review of draft manuscript, and approval of final version; T.M. contributed to the acquisition of data, analysis of data, interpretation of data, review of draft manuscript, and approval of final version; R.S. contributed to the conception/design of the study, acquisition of data, analysis of data, interpretation of data, review of draft manuscript, and approval of final version; J.W.V. contributed to the conception/design of the study, acquisition of data, analysis of data, interpretation of data, review of draft manuscript, and approval of final version. All authors had full access to all the data in the study and had final responsibility for the decision to submit for publication.

\section{References}

1 Kunz PL. Understanding Neuroendocrine Tumors-A NET Gain. JAMA Oncol. 2017 Oct;3(10):1343-4.

2 Dasari A, Shen C, Halperin D, Zhao B, Zhou $\mathrm{S}, \mathrm{Xu} \mathrm{Y}$, et al. Trends in the Incidence, Prevalence, and Survival Outcomes in Patients With Neuroendocrine Tumors in the United States. JAMA Oncol. 2017 Oct;3(10):133542.

3 Barakat MT, Meeran K, Bloom SR. Neuroendocrine tumours. Endocr Relat Cancer. 2004 Mar;11(1):1-18.
4 Bosman FT, Carneiro F, Hruban RH, Theise ND. WHO Classification of Tumours, Volume 3 IARC WHO Classification of Tumours, No 3 IARC; 2010.

5 Chai SM, Brown IS, Kumarasinghe MP. Gastroenteropancreatic neuroendocrine neoplasms: selected pathology review and molecular updates. Histopathology. 2018 Jan;72(1): 153-67.

6 Lloyd RV, Osamura RY, Klöppel G, Rosai J. WHO Classification of Tumours of Endocrine Organs. 2017 [cited 2018 Oct 9]. Available from: http://publications.iarc.fr/Book-AndReport-Series/Who-Iarc-Classification-OfTumours/Who-Classification-Of-TumoursOf-Endocrine-Organs-2017.

7 National Institute for Health and Care Excellence. Everolimus and sunitinib for treating unresectable or metastatic neuroendocrine tumours in people with progressive disease (TA449). 2017 [cited 2017 Dec 7]. Available from: https://www.nice.org.uk/ guidance/ta449/chapter/1-Recommendations. 
8 Raymond E, Dahan L, Raoul JL, Bang YJ, Borbath I, Lombard-Bohas C, et al. Sunitinib malate for the treatment of pancreatic neuroendocrine tumors. N Engl J Med. 2011 Feb; 364(6):501-13.

9 Yao JC, Shah MH, Ito T, Bohas CL, Wolin EM, Van Cutsem E, et al.; RAD001 in Advanced Neuroendocrine Tumors, Third Trial (RADIANT-3) Study Group. Everolimus for advanced pancreatic neuroendocrine tumors. N Engl J Med. 2011 Feb;364(6):514-23.

10 Swinburn P, Wang J, Chandiwana D, Mansoor W, Lloyd A. Elicitation of health state utilities in neuroendocrine tumours. J Med Econ. 2012;15(4):681-7.

11 Pezzilli R, Campana D, Morselli-Labate AM, Fabbri MC, Brocchi E, Tomassetti P. Patientreported outcomes in subjects with neuroendocrine tumors of the pancreas. World J Gastroenterol. 2009 Oct;15(40):5067-73.
12 Chau I, Casciano R, Willet J, Wang X, Yao JC. Quality of life, resource utilisation and health economics assessment in advanced neuroendocrine tumours: a systematic review. Eur J Cancer Care (Engl). 2013 Nov;22(6):714-25.

13 Novartis Pharmaceuticals UK. Ltd: Afinitor (everolimus) summary of product characteristics 2017 [cited 2017 Dec 7]. Available from: https://www.medicines.org.uk/emc/medicine/22281.

14 Aaronson NK, Ahmedzai S, Bergman B, Bullinger M, Cull A, Duez NJ, et al. The European Organization for Research and Treatment of Cancer QLQ-C30: a quality-of-life instrument for use in international clinical trials in oncology. J Natl Cancer Inst. 1993 Mar;85(5):365-76.

15 Davies AHG, Larsson G, Ardill J, Friend E, Jones L, Falconi M, et al. Development of a disease-specific Quality of Life questionnaire module for patients with gastrointestinal neuroendocrine tumours. Eur J Cancer. 2006 Mar;42(4):477-84.
16 Cheung YB, Thumboo J, Gao F, Ng GY, Pang G, Koo WH, et al. Mapping the English and Chinese versions of the Functional Assessment of Cancer Therapy-General to the EQ5D utility index. Value Health. 2009 MarApr;12(2):371-6.

17 Pavel M, Unger N, Borbath I, Ricci S, Hwang TL, Brechenmacher T, et al. Safety and QOL in Patients with Advanced NET in a Phase $3 \mathrm{~b}$ Expanded Access Study of Everolimus. Target Oncol. 2016 Oct;11(5):667-75.

18 Beaumont JL, Cella D, Phan AT, Choi S, Liu Z, Yao JC. Comparison of health-related quality of life in patients with neuroendocrine tumors with quality of life in the general US population. Pancreas. 2012 Apr; 41(3):461-6. 